\title{
Bivalve Tropomyosins Exhibit Strong Inhibition of Actomyosin Mg-ATPase and High Viscosity
}

\author{
Yasushi Nishimura, ${ }^{\dagger 1}$ Takao Ojima, and Kiyoyoshi Nishita ${ }^{\dagger 2}$ \\ Department of Chemistry, Faculty of Fisheries, Hokkaido University, Hakodate, Hokkaido 041, Japan \\ (Received December 16, 1996)
}

\begin{abstract}
Tropomyosins were isolated from striated and smooth adductor muscles of akazara scallop Chlamys nipponensis akazara and ezo-giant scallop Patinopecten yessoensis and smooth adductor muscle of surf clam Spisula sacharinensis. These bivalve tropomyosins inhibited Mg-ATPase activity of rabbit reconstituted actomyosin to $15 \%$ of original activity, i.e., approximately 1.4 times stronger than rabbit and carp tropomyosins did at a weight ratio of 2:1 for F-actin: tropomyosin. On the other hand, the relative viscosities in the absence of $\mathrm{KCl}$ were 3.5-6 times higher than those of rabbit and carp tropomyosins. The intrinsic viscosities were, however, comparable to that of rabbit tropomyosin, i.e., $0.5 \mathrm{~d} l / \mathrm{g}$ for bivalve tropomyosins and $0.4 \mathrm{~d} / / \mathrm{g}$ for rabbit tropomyosin. These viscosities suggest that the bivalve tropomyosins tend to form a highly polymerized form compared with rabbit tropomyosin. Circular dichroism spectra and amino acid compositions of the bivalve tropomyosins are similar to one another and also to rabbit and carp tropomyosins. C-terminal three amino acids of bivalve tropomyosins were sequenced as -Ala-Gly-Tyr (scallops) and-Gly-Tyr-Thr (surf clam), which are quite different from -Thr-Ser-Ile and Thr-Ser-Leu of rabbit $\alpha$ - and $\beta$-tropomyosin, respectively.
\end{abstract}

Key words: bivalve, tropomyosin, inhibition of Mg-ATPase, viscosity

Tropomyosin is a well-investigated regulatory protein in muscle and non-muscle cells. ${ }^{1-7)}$ It is a highly $\alpha$-helical, rod-shaped dimeric protein forming a coiled-coil structure and binds along the grooves of F-actin filaments. In striated muscles and some of smooth muscles, tropomyosin plays a regulatory role for contraction together with troponin. ${ }^{7-10)}$ Up to now a number of tropomyosin isoforms have been isolated from various vertebrate and invertebrate muscles and the molecular heterogeneity and tissue specificity have been extensively investigated. ${ }^{2,6,11-15)}$

On the other hand, we have isolated tropomyosin and troponin from striated and smooth adductor muscles of both akazara scallop and ezo-giant scallop in which a myosin-linked regulatory system exists. We have also shown that those tropomyosins strongly inhibit Mg-ATPase activity of rabbit reconstituted actomyosin..$^{9,10,16,17)}$ It is considered that scallop troponin can confer $\mathrm{Ca}^{2+}$-sensitivity to actomyosin in such a manner as increasing the repressed activity by tropomyosin in the presence of $\mathrm{Ca}^{2+}$ rather than decreasing the activity in its absence as seen in vertebrate regulatory systems. A similar conferring manner of $\mathrm{Ca}^{2+}$. sensitivity has also been reported in ascidian smooth muscle tropomyosin-troponin. ${ }^{8)}$ Thus, the strong inhibitory effect of tropomyosin on actomyosin Mg-ATPase seems to be a characteristic property of invertebrate tropomyosins.

In the present paper, we show that several bivalve tropomyosins also exhibit strong inhibitory effects on rabbit actomyosin Mg-ATPase activity, in addition to extremely high specific viscosity.

\section{Materials and Methods}

Tropomyosin was prepared from striated and smooth adductor muscles of akazara scallop and ezo-giant scallop and smooth adductor muscle of surf clam. Preparation was achieved by the methods described by Endo and

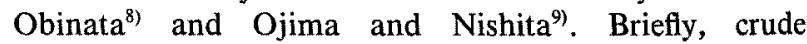
tropomyosin was extracted with $0.4 \mathrm{M} \mathrm{LiCl}$ at $\mathrm{pH} 5.0$ from the myofibrils and purified by repeating ammonium sulfate fractionation at $35-45 \%$ saturation in the presence of $0.4 \mathrm{M} \mathrm{LiCl}$. Rabbit and carp tropomyosins were isolated from white skeletal muscle and dorsal muscle, respectively, by the method used for the scallop tropomyosins except that the ammonium sulfate fractionation was achieved at $45-55 \%$ saturation. Rabbit myosin and actin were prepared by the methods of Perry ${ }^{18)}$ and Spudich and Watt, ${ }^{19)}$ respectively. Actomyosin was reconstituted by mixing the myosin and actin in a weight ratio of 2:1. SDSPAGE was performed by the method of Porzio and Pearson. ${ }^{20)}$ The gel was stained with $0.2 \%$ Coomassie Brilliant Blue R-250 in 50\% methanol and 10\% acetic acid, and background of the gel was destained with $10 \%$ acetic acid and $5 \%$ methanol. Mg-ATPase activity of actomyosin was measured in a solution containing $50 \mathrm{mM} \mathrm{KCl}, 20 \mathrm{~mm}$ Tris maleate ( $\mathrm{pH} 6.8$ ), $2 \mathrm{mM} \mathrm{MgCl}_{2}, 0.1 \mathrm{mg} / \mathrm{m} / \mathrm{rabbit}$ myosin, $0.05 \mathrm{mg} / \mathrm{m} l \mathrm{rab}$ it $\mathrm{F}$-actin, and various concentrations of tropomyosin. The inorganic phosphate liberated by ATP hydrolysis was determined by the method of Youngburg

\footnotetext{
1. Present address: Ajinomoto Co., Inc., Suzuki, Kawasaki, Kawasaki 210, Japan.

†2 To whom correspondence should be addressed.

Abbreviations: SDS, sodium dodecyl sulfate; PAGE, polyacrylamide gel electrophoresis; Mr, molecular weight.
} 
and Youngburg. ${ }^{21)}$ The viscosity of tropomyosin was measured with Ostwald's viscometer (flow time of the solvent was $29 \mathrm{~s}$ ). Circular dichroism spectrum was measured by using a Jasco J-600 spectropolarimeter and a cuvette with a $1 \mathrm{~mm}$ path length. The $\alpha$-helical content was estimated with the following equation:22)

$$
\text { helix }(\%)=\frac{[\theta]_{222}+3,000}{39,000} \times 100 .
$$

Amino acid composition was analyzed by using a Hitachi 655-A high performance liquid chromatograph equipped with Wakosil-PTC column $(4 \times 200 \mathrm{~mm})$. The amino acid sequence of the $\mathrm{C}$-terminal region was determined by the digestion of carboxypeptidase $\mathrm{Y}$ followed by the analysis of released amino acids. Protein concentration was determined by the biuret method using bovine serum albumin Fraction $\mathrm{V}$ as a standard protein. ${ }^{23)}$

\section{Results and Discussion}

\section{SDS-PAGE of Bivalve Tropomyosin}

Figure 1 shows the SDS-gel electrophoretic patterns of seven species of tropomyosin. Striated adductor tropomyosins from both akazara and ezo-giant scallops showed a single band of around Mr 35,000, while smooth adductor tropomyosins showed two subunit bands with $\mathrm{Mr} 35,000$ and 36,000. The surf clam smooth muscle tropomyosin showed a single band with $\mathrm{Mr} 34,000$. These results coincide with the previous reports..$^{11,14,24)}$ Thus, two isoforms and one isoform with respect to the mobility on SDSPAGE were observed in scallop smooth and striated muscle tropomyosins, respectively, ${ }^{14,24)}$ while no polymor-

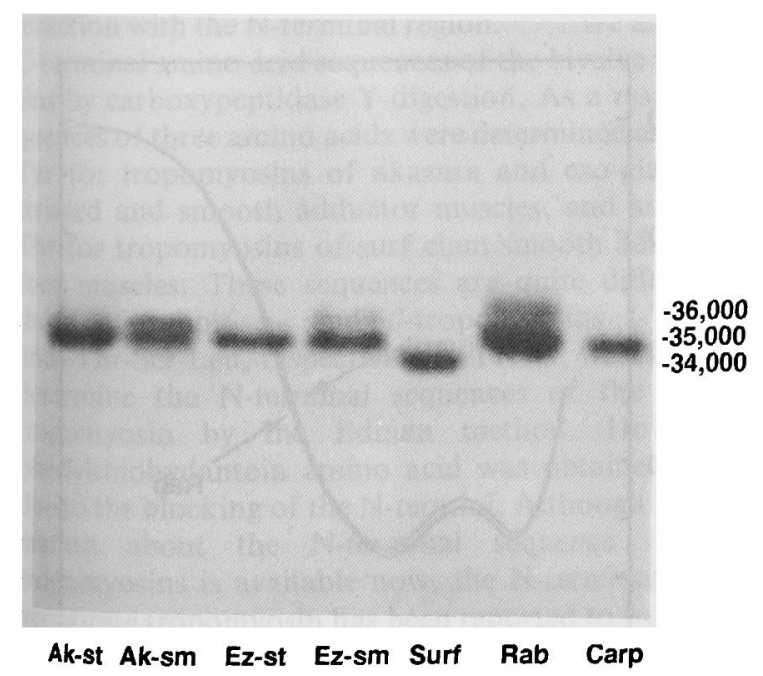

Pig. 1. SDS-polyacrylamide gel electrophoresis of various tropomyosins.

Electrophoresis was carried out in $10 \%$ polyacrylamide slab gel containing $0.1 \%$ SDS according to Porzio and Pearson. ${ }^{20)}$ Ak-st, akazara scallop striated adductor Tm; Ak-sm, akazara scallop smooth adductor Tm; Ez-st, ezo-giant scallop striated adductor Tm; Ez-sm, ezo-giant scallop smooth adductor Tm; Surf, surf clam smooth adductor Tm, Rab, rabbit skeletal Tm; Carp, carp dorsal muscle $\mathrm{Tm}$. The approximate molecular weights are shown in the right-hand side of the figure. phism was seen in surf clam tropomyosin. ${ }^{11)}$ The rabbit and carp tropomyosins showed a doublet and singlet band, respectively, as reported previously. ${ }^{7,25,26)}$

\section{Effects of Bivalve Tropomyosins on Actomyosin $\mathrm{Mg}-\mathrm{AT}$ - Pase Activity}

We have shown that tropomyosin from scallop striated and smooth muscles strongly inhibits Mg-ATPase activity of rabbit reconstituted actomyosin, and the scallop troponin confers $\mathrm{Ca}^{2+}$-sensitivity to the actomyosin-tropomyosin Mg-ATPase activity by removing the inhibition of tropomyosin in the presence of $\left.\mathrm{Ca}^{2+} .9,10,16,17\right)$ Therefore, we examined the inhibitory effect of bivalve tropomyosins on the actomyosin Mg-ATPase activity and compared with those of rabbit and carp tropomyosins. As shown in Fig. 2 , all the bivalve tropomyosins tested inhibited approximately $85 \%$ of original Mg-ATPase activity, while the rabbit and carp tropomyosins inhibited $60 \%$. Thus, those tropomyosins appear to be classified into two types, i.e., one showing stronger inhibition (bivalve tropomyosins) and the other showing weaker inhibition (rabbit and carp tropomyosins). The physiological significance of the strong inhibition of the bivalve tropomyosins is obscure. However, it has been reported that superprecipitation activity and its $\mathrm{Ca}^{2+}$-sensitivity of ezo-giant scallop striated adductor actomyosin are improved by the presence of scallop tropomyosin. ${ }^{24)}$ The effects of coexistence of myosinlinked regulatory system on the function of bivalve tropomyosins are now under investigation.

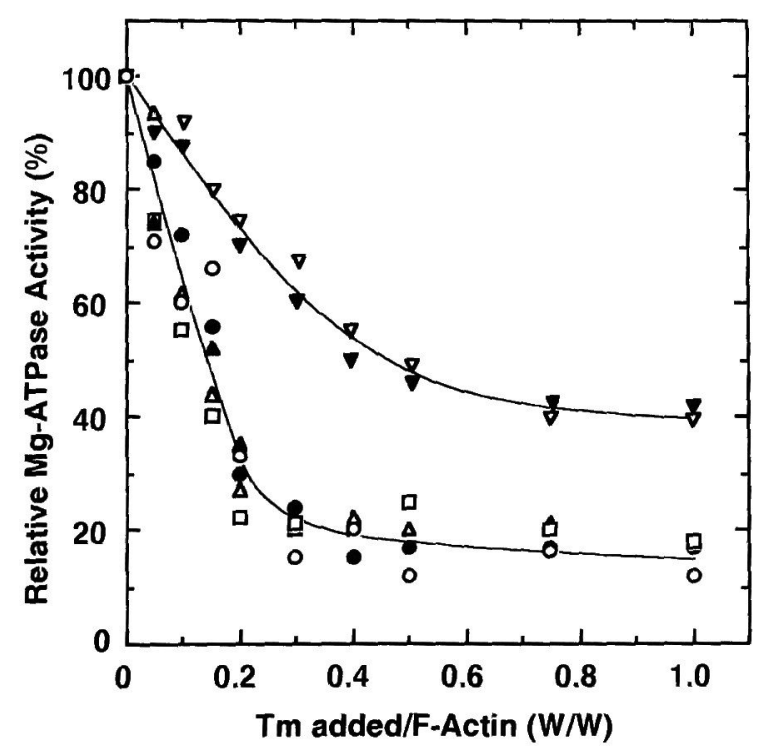

Fig. 2. Effect of tropomyosin on the Mg-ATPase activity of rabbit reconstituted actomyosin.

Mg-ATPase activity was measured as described in "Materials and Methods". O and $\bullet$, akazara scallop striated and smooth adductor Tms, respectively; $\Delta$ and $\boldsymbol{\Delta}$, ezo-giant scallop striated and smooth adductor Tms, respectively; $\square$, surf clam smooth adductor Tm; $\nabla$, rabbit $\mathrm{Tm} ; \boldsymbol{\nabla}$, carp $\mathrm{Tm}$. Activity of actomyosin in the absence of $\mathrm{Tm}$ was $0.098 \mu \mathrm{mol} \mathrm{Pi} / \mathrm{min} \cdot \mathrm{mg}$ and represented as $100 \%$ relative activity on the ordinate. 
Viscosity of Bivalve Tropomyosins

The head-to-tail polymerization ability of tropomyosin has been considered as a crucially important function for the $\mathrm{Ca}^{2+}$-regulation of troponin-tropomyosin. ${ }^{7,27,28)}$ Since the polymerization of tropomyosin is enhanced under lower ionic conditions, the viscosity becomes higher concomitantly with decrease in salt concentration. As shown in Fig. 3, the relative viscosity steeply increased with decrease in $\mathrm{KCl}$ concentration below $0.05 \mathrm{M}$. In addition, the extent of increase in viscosity of bivalve tropomyosins was higher than those of rabbit and carp tropomyosins. The relative viscosities of striated and smooth muscle tropomyosins of akazara and ezo-giant scallops were about 6 times higher than those of rabbit and carp tropomyosins in the absence of $\mathrm{KCl}$, and that of surf clam Tm was about 3.5 times higher than those of rabbit and carp tropomyosins. The reduced viscosities of bivalve tropomyosins were plotted against concentration along with those of rabbit and carp (Fig. 4). Accordingly, the intrinsic viscosities of the bivalve tropomyosins were estimated to be $0.5 \mathrm{~d} l / \mathrm{g}$, slightly higher than those of rabbit and carp tropomyosins of $0.4 \mathrm{~d} l / \mathrm{g}$. These results strongly suggest that the bivalve tropomyosins have markedly higher polymerizability than vertebrate tropomyosins at lower salt concentrations, though their molecular dimensions appear to be similar.

\section{Circular Dichroism of Bivalve Tropomyosins}

The tropomyosin molecule is known to be formed from almost a complete $\alpha$-helix structure. ${ }^{7,22)}$ As shown in Fig. 5 , the circular dichroism spectra of all the tropomyosins tested were indistinguishable from one another. Their $\alpha$ helical contents were estimated to be about $85 \%$ from the

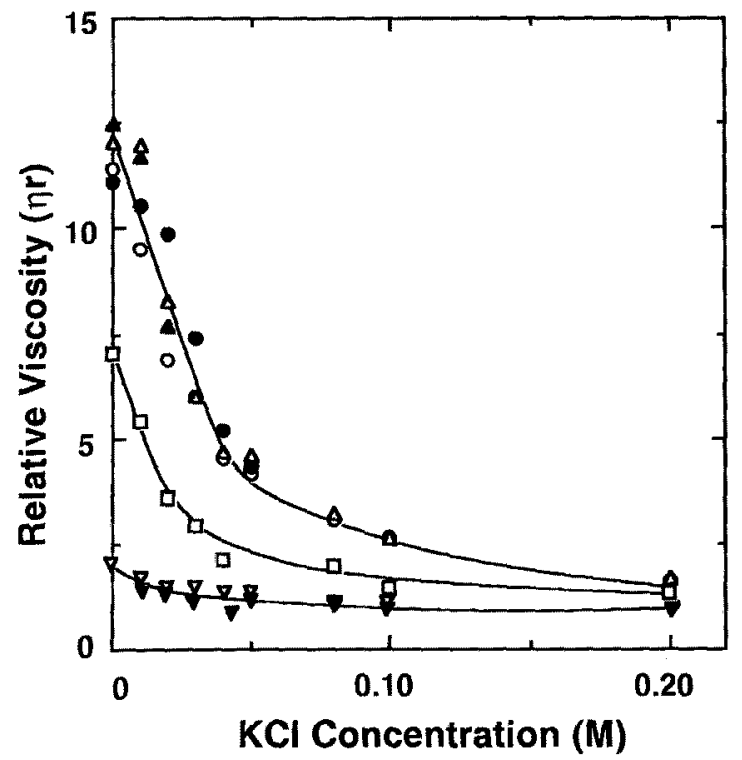

Fig. 3. Relative viscosity of tropomyosin as a function of $\mathrm{KCl}$ concentration.

The relative viscosity was determined at $20^{\circ} \mathrm{C}$ in an Ostwald type viscometer with the outflow time of $29.0 \mathrm{~s}$ for solvent. The concentration of $\mathrm{Tm}$ was $1.5 \mathrm{mg} / \mathrm{m} l$ in $20 \mathrm{~mm}$ Tris maleate $(\mathrm{pH} \mathrm{7.0)}$ and various concentrations of $\mathrm{KCl}$. The symbols used are the same as in Fig. 2. molecular ellipticity at $222 \mathrm{~nm}$. No difference in $\alpha$-helical content was found among the tropomyosins examined.

Amino Acid Compositions of the Bivalve Tropomyosins Amino acid compositions of tropomyosins were deter-

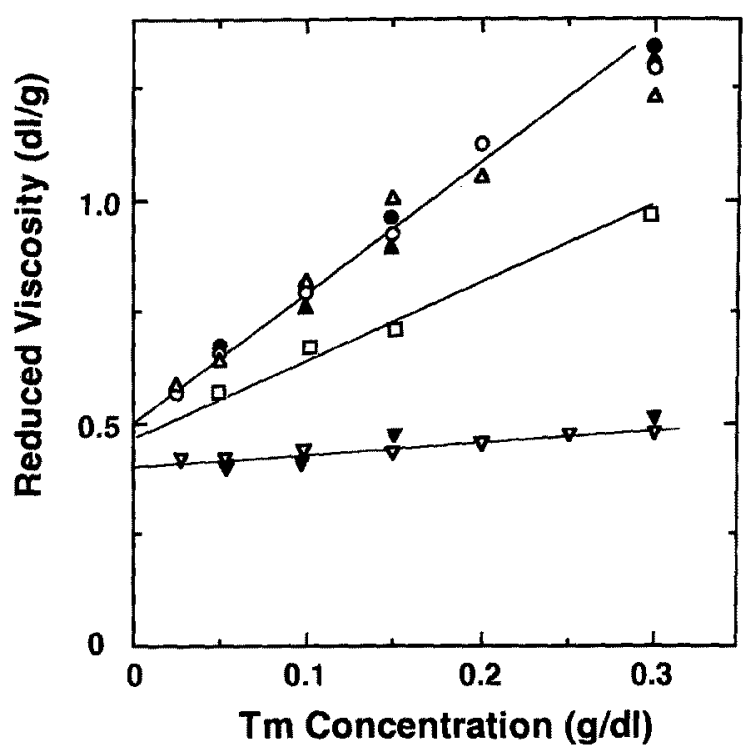

Fig. 4. Reduced viscosity of tropomyosin as a function of protein concentration.

Viscosity was measured as in Fig. 3, except that the $\mathrm{KCl}$ concentration was fixed to $200 \mathrm{~mm}$ and the protein concentration was varied. 0 , akazara scallop striated adductor $\mathrm{Tm} ; \triangle$, ezo-giant scallop striated adductor Tm; $\boldsymbol{\Delta}$, surf clam smooth adductor $\mathrm{Tm} ; \nabla$, rabbit $\mathrm{Tm}$.

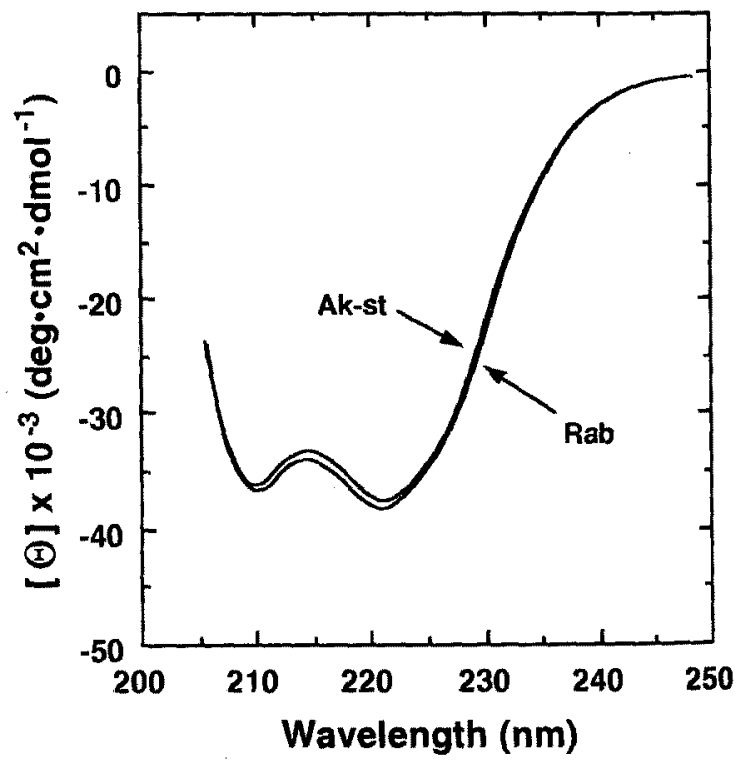

Fig. 5. CD-spectrum of tropomyosin.

The CD-spectrum was measured in a solution containing $0.1 \mathrm{M}$ $\mathrm{KCl}, 10 \mathrm{mM}$ Tris-HCl (pH 7.5), $1 \mathrm{mg} / \mathrm{ml} \mathrm{Tm}$. The spectra for Tms of akazara scallop striated adductor (Ak-st) and rabbit (Rab) are shown as representatives since the profiles of the other Tms were vractically identical. 
Table 1. Amino acid composition of various tropomyosins (mol\%)

\begin{tabular}{|c|c|c|c|c|c|c|c|}
\hline $\begin{array}{l}\text { Amino } \\
\text { acid }\end{array}$ & Ak-st & Ak-sm & Ez-st & $\mathrm{Ez}-\mathrm{sm}$ & Surf & Rab & Carp \\
\hline Asx*1 & 13.2 & 13.6 & 13.9 & 14.0 & 11.2 & 10.8 & 11.1 \\
\hline Thr & 7.1 & 5.0 & 7.1 & 4.1 & 5.6 & 1.9 & 3.5 \\
\hline Ser & 3.3 & 3.7 & 3.9 & 3.4 & 5.1 & 4.5 & 3.8 \\
\hline $\mathrm{Glx}^{*_{2}}$ & 26.0 & 26.0 & 24.7 & 26.8 & 22.6 & 26.7 & 25.9 \\
\hline Gly & 1.8 & 2.0 & 1.7 & 2.0 & 1.8 & 1.6 & 1.9 \\
\hline Ala & 11.3 & 12.9 & 11.5 & 11.9 & 10.3 & 12.7 & 12.4 \\
\hline $\mathrm{Val}$ & 4.0 & 3.6 & 4.4 & 4.3 & 5.7 & 3.1 & 3.1 \\
\hline Met & 1.1 & 0.5 & 1.5 & 0.6 & 1.4 & 1.3 & 2.3 \\
\hline Ile & 2.3 & 3.5 & 2.8 & 3.7 & 4.4 & 4.8 & 3.6 \\
\hline Leu & 10.8 & 11.5 & 10.6 & 11.5 & 11.7 & 11.3 & 11.3 \\
\hline Tyr & 1.1 & 1.3 & 1.2 & 0.4 & 2.6 & 1.4 & 1.5 \\
\hline Phe & 1.8 & 1.5 & 1.3 & 1.9 & 0.6 & 0.9 & 0.9 \\
\hline Lys & 10.1 & 7.9 & 9.9 & 8.9 & 10.6 & 13.4 & 13.0 \\
\hline His & 0.4 & 0.4 & 0.0 & 0.2 & 0.0 & 0.6 & 0.8 \\
\hline Arg & 6.1 & 6.6 & 5.5 & 6.3 & 6.4 & 5.0 & 4.9 \\
\hline Pro & 0.0 & 0.0 & 0.0 & 0.0 & 0.0 & 0.0 & 0.0 \\
\hline Total & 100.0 & 100.0 & 100.0 & 100.0 & 100.0 & 100.0 & 100.0 \\
\hline
\end{tabular}

Abbreviations are the same as in Fig. 1.

${ }^{*}$ Asp + Ass. ${ }^{*}$ Glu + Gln.

mined (Table 1). All tropomyosins tested showed fairly similar compositions, i.e., rich in Asx, Glx, Ala, Leu, and Lys, but no Pro. The absence of Pro is a common feature of tropomyosins. ${ }^{29-33)}$ However, some differences were seen, for example, the bivalve tropomyosins contained more $\mathrm{Thr}$ and less $\mathrm{Lys}^{26,29,30)}$ than rabbit and carp tropomyosins.

\section{Amino Acid Sequence of C-terminal Region of Bivalve Tropomyosins}

In rabbit skeletal tropomyosin, the C-terminal 9 amino acids have been shown to be involved in the head-to-tail interaction with the $\mathrm{N}$-terminal region. ${ }^{7,27,28)}$ We analyzed the C-terminal amino acid sequences of the bivalve tropomyosins by carboxypeptidase $Y$ digestion. As a result, the sequences of three amino acids were determined as -Ala-GlyTyr for tropomyosins of akazara and ezo-giant scallop striated and smooth adductor muscles, and as -Gly-TyrThr for tropomyosins of surf clam smooth adductor and foot muscles. These sequences are quite different from those of rabbit $\alpha$ - and $\beta$-tropomyosins (-Thr-Ser-Ile and -Thr-Ser-Leu, respectively ${ }^{31,32)}$ ) Next, we attempted to determine the $\mathrm{N}$-terminal sequences of the molluscan tropomyosin by the Edman method. However, no phenylthiohydantoin amino acid was obtained probably due to the blocking of the $\mathrm{N}$-termini. Although little information about the $\mathrm{N}$-terminal sequence of bivalve tropomyosins is available now, the $\mathrm{N}$-terminal region of vertebrate tropomyosin has been reported to be fairly conserved among various species in contrast to the divergence in the C-terminal region. ${ }^{33)}$ In order to reveal the reason for the high viscosity of bivalve tropomyosins, detailed studies on the primary structures and polymerizabilities are necessary.

\section{References}

1) K. Bailey: Tropomyosin: a new asymmetric protein of the muscle fibril. Biochem. J., 43, 271-278 (1948).
2) S. Ebashi: Regulation of the myosin-actin interaction by the $\mathrm{Ca}^{2+}$ troponin-tropomyosin system. J. Biochem., 79, 48-49 (1976).

3) J. Hayashi, T. Ishimoda, and T. Hirabayashi: On the heterogeneity and organ specificity of chicken tropomyosins. J. Biochem., 81, 1487-1495, (1977).

4) T. Ishimoda-Takagi: Localization of tropomyosin in sea urchin eggs. Exp. Cell Res., 119, 423-428 (1979).

5) G. P. Cote and L. B. Smillie: Preparation and some properties of equine platelet tropomyosin. J. Biol. Chem., 256, 11004-11010 (1981).

6) G. P. Cote: Structural and functional properties of the non-muscle tropomyosins. Mol. Cell. Biochem., 57, 127-146 (1983).

7) I. Ohtsuki, K. Maruyama, and S. Ebashi: Regulatory and cytoskeletal proteins of vertebrate skeletal muscle. Adv, Prot. Chem., 38, 1-67 (1986).

8) T. Endo and T. Obinata: Troponin and its components from ascidian smooth muscle. J. Biochem., 89, 1599-1608 (1981).

9) T. Ojima and K. Nishita: Isolation of troponins from striated and smooth adductor muscles of akazara scallop. J. Biochem., 100, 821-824 (1986).

10) K. Nishita , T. Ojima, A. Takahashi, and A. Inoue: Troponin from smooth adductor muscle of ezo-giant scallop. J. Biochem., 121, 419-424 (1997).

11) B. A. Margulis and G. P. Pinaev: The species specificity of the contractile protein composition of the bivalve molluscs. Comp. Biochem. Physiol., 55B, 189-194 (1976).

12) G. K. Dhoot and S. V. Perry: Distribution of polymorphic forms of troponin components and tropomyosin in skeletal muscle. Nature, 278, 714-718 (1979).

13) D. D. Bronson and F. H. Schachat: Heterogeneity of contractile proteins. J. Biol. Chem., 257, 3937-3944 (1982).

14) T. Ishimoda-Takagi and M. Kobayashi: Molecular heterogeneity and tissue specificity of tropomyosin obtained from various bivalves. Comp. Biochem. Physiol., 88B, 443-452 (1987).

15) D. H. Heeley and C. Hong: Isolation and characterization of tropomyosin from fish muscles. Comp. Biochem. Physiol., 108B, 95-106 (1994)

16) T. Ojima and K. Nishita: Troponin from akazara scallop striated adductor muscles. J. Biol. Chem., 261, 16749-16754 (1986).

17) T. Ojima and K. Nishita: Comparative studies on biochemical characteristics of troponins from ezo-giant scallop (Patinopecten yessoensis) and akazara scallop (Chlamys nipponensis akazara). Comp. Biochem. Physiol, 103B, 727-732 (1992).

18) S. V. Perry: Myosin adenosinetriphosphatase, in "Methods in En- 
zymology" (ed. by S. P. Colowick and N. O. Kaplan) Vol. 2, Academic Press, New York, 1955, pp. 582-588.

19) J. A. Spudich and S. Watt: The regulation of rabbit skeletal muscle contraction: I. Biochemical studies of the interaction of the tropomyosin-troponin complex with actin and the proteolytic fragment of myosin. J. Biol. Chem., 246, 4866-871 (1971).

20) M. A. Porzio and A. M. Pearson: Improved resolution of myofibrillar proteins with sodium dodecyl sulfate-polyacrylamide gel electrophoresis. Biochim. Biophys. Acta, 490, 27-34 (1977).

21) G. E. Youngburg and M. N. Youngburg: Phosphorus metabolism 1. A system of blood phosphorus analysis. J. Lab. Clin. Med., 16, $158-166(1930)$.

22) C. S. Wu and J. T. Yang: Reexamination of the conformation of muscle proteins by optical activity. Biochemistry, 15, 3007-3014 (1976).

23) A. G. Gornall, C. S. Bardawill, and M. N. David: Determination of serum proteins by means of the biuret reaction. J. Biol. Chem., 177, 751-766 (1949).

24) M. Takahashi and F. Morita: An activating factor (tropomyosin) for the superprecipitation of actomyosin prepared from scallop adductor muscles. J. Biochem., 99, 339-347 (1986).

25) N. Seki and S. Iwabuchi: On the subunit composition of fish tropomyosin. Nippon Suisan Gakkaishi, 44, 1333-1340 (1978),

26) N. Ookubo, H. Ueno, and T. Ooi: Similarities and differences of the alpha and beta components of tropomyosin. J. Biochem., 78, 739-747 (1975).

27) P. Johnson and L. B. Smillie: Polymerizability of rabbit skeletal tropomyosin: effects of enzymic and chemical modifications. Biochemistry, 16, 2264-2269 (1977).

28) H. Ueno, Y. Tawada, and T. Ooi: Properties of non-polymerizable tropomyosin obtained by carboxypeptidase A digestion. $J$. Biochem., 80, 283-290 (1976).

29) E. F. Woods and M. J. Pont : Characterization of some invertebrate tropomyosins. Biochemistry, 10, 270-276 (1971).

30) R. E. Fine and A. L. Blitz: A chemical comparison of tropomyosins from muscle and non-muscle tissues. J. Mol. Biol., 95, 447-454 (1975).

31) D. Stone and L. B. Smillie: The amino acid sequence of rabbit

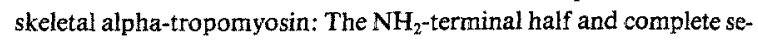
quence. J. Biol. Chem., 253, 1137-1148 (1978).

32) A. S. Mak, L. B. Smillie, and G. R. Steward: A comparison of the amino acid sequences of rabbit skeletal muscle alpha- and betatropomyosins. J. Biol. Chem., 255, 3647-3655 (1980).

33) D. M. Helfman, J. R. Feramisco, W. M. Ricci, and S. H. Hughes: Isolation and sequence of a cDNA clone that contains the entire coding region for chicken smooth-muscle $\alpha$-tropomyosin. $J$. Biol. Chem., 259, 14136-14143 (1984). 